investigating the roles of these ligands and their receptors in the $\mathrm{K} / \mathrm{BxN}$ mouse model of RA, Wu et al. surprisingly found that some oligodeoxynucleotides (ODNs) carrying unmethylated CpG motifs inhibit the development of RA.

In the $\mathrm{K} / \mathrm{BxN}$ model, serum transferred from transgenic mice (which spontaneously develop RA) induces RA in nontransgenic animals. Although peptidoglycans and lipopolysaccharides exacerbated RA development when injected into mice before serum transfer, certain CpG ODNs inhibited development of RA. ODN 1668 severely inhibited RA development when administered before or at the same time as the inflammatory serum injection. This molecule also had a therapeutic effect on pre-existing arthritis; although ODN 1668 administration did not reverse RA symptoms, it prevented worsening of the disease. The inhibitory effect of ODN 1668 was mediated through binding to Toll-like receptor TLR9 on CD8 $\alpha^{+}$dendritic cells. Interleukin-12 produced by these cells stimulated natural killer cells to make interferon- $\gamma$, which inhibited RA development by preventing recruitment of neutrophils to the inflamed joint.

The findings suggest a possible therapeutic use of CpG ODNs as anti-inflammatory agents.

Original article Wu HJ et al. (2007) Inflammatory arthritis can be reined in by CpG-induced DC-NK cell cross talk. J Exp Med 204: 1911-1922

\section{Novel helper T cell clones from patients with Crohn's disease}

T helper $17\left(\mathrm{~T}_{\mathrm{H}} 17\right)$ cells are a novel subset of helper $\mathrm{T}$ cells, named for their ability to produce interleukin (IL)-17, that are associated with autoimmune diseases in mice. Researchers in Italy have isolated $T_{H} 17$ cells from the gut of patients with Crohn's disease. A large proportion of the isolated cells produced both IL-17 and interferon- $\gamma$, which is normally produced by $T_{H} 1$ cells, and were therefore termed

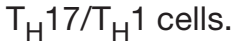

Both $T_{H^{17}} 17 / T_{H} 1$ and $T_{H} 17$ cells facilitated the production by $B$ cells of $\lg G$, IgM and $\lg A$ antibodies, but not IgE production. Both cell types had low cytotoxic capability, with poor granzyme A production. $T_{H} 17 / T_{H} 1$ and $T_{H} 17$ cells selectively expressed IL-23R, CCR6 and the transcription factors ROR $\gamma \mathrm{t}$ and T-bet (also known as TBX21). Stimulation with IL-12 induced $T_{H} 17$ cells to produce interferon- $\gamma$, increased T-bet expression (both these molecules are associated with $\mathrm{T}_{\mathrm{H}} 1$ cells) and downregulated ROR $\gamma \mathrm{t}$ and IL-17, but these effects could be partly inhibited by IL-23. IL-17-producing cells were detected not only in Crohn's disease tissue, but also in normal gut tissue, peripheral blood and tonsils.

The authors conclude that $\mathrm{T}_{\mathrm{H}} 17$ cells display distinct properties of $\mathrm{T}_{\mathrm{H}} 1$ and $\mathrm{T}_{\mathrm{H}} 2$ cells, but the discovery of $\mathrm{T}_{\mathrm{H}} 17 / \mathrm{T}_{\mathrm{H}} 1$ cells, and their modulation by IL-12, suggests a functional or developmental relationship between $\mathrm{T}_{\mathrm{H}} 17$ and $\mathrm{T}_{\mathrm{H}} 1$ cells.

Original article Annunziato F et al. (2007) Phenotypic and functional features of human Th17 cells. J Exp Med 204: 1849-1861

\section{SLE can develop independently of $\mathbf{T}$ cells}

B cells are well-established as central players in the pathogenesis of systemic lupus erythematosus (SLE). T cells are also thought to have an important role, since inhibition of $\mathrm{T}$-cell activation and $\mathrm{T}$ cell-B cell interactions prevent autoimmunity in animal models of SLE. B cell-activating factor of the tumor necrosis factor family (BAFF) is essential for the maturation and survival of peripheral $B$ cells. BAFF is also a co-stimulatory factor for $\mathrm{T}$ cells and promotes differentiation of T-cells to effector cells. Levels of BAFF are elevated in patients with SLE, and mice overexpressing BAFF (BAFF Tg mice) exhibit symptoms of lupus-like disease. New data indicates that BAFF induces SLE-like disease in a T-cell-independent manner.

BAFF Tg mice exhibit expanded B-cell and T-cell compartments, as well as excessive autoantibody production. Groom et al. studied the effects of $T$ cells on BAFF-mediated lupus by comparing these BAFF Tg mice with mice expressing the same high levels of BAFF, but completely lacking $\alpha / \beta$ and $\gamma / \delta$ T cells (TA-BTg mice). BAFF-mediated expansion of the B-cell compartment, increases in serum levels of IgG2c, IgG2b, IgG3 and IgM, as well as production of SsDNA, dsDNA, and rheumatoid factor autoantibodies, nephritis, and autoimmune pathology such as salivary gland destruction and infiltration by marginal zone $B$ cells, were all found to be T-cell-independent 